Recibido: 27/04/2019 --- Aceptado: 05/08/2019 --- Publicado: 15/03/2020

\title{
TRADICIÓN E INNOVACIÓN EN TWITTER. UN ENSAYO DE TRADICIONES DISCURSIVAS EN CUENTAS MEXICANAS A TRAVÉS DE LA JUNCIÓN
}

\section{TRADITION AND INNOVATION IN TWITTER. AN ESSAY OF DISCOURSE TRADITIONS IN MEXICAN ACCOUNTS THROUGH JUNCTION}

Juan Ricardo Ubiarco Moya: El Colegio de México A. C. México. jubiarco@colmex.mx

\section{RESUMEN}

Estas líneas presentan algunos resultados obtenidos sometiendo a la experimentación una hipótesis que sugiere la posibilidad de identificar síntomas de una Tradición Discursiva -entendida como todo molde históricamente determinado para la comunicación en una lengua con su propia historicidad- en un texto a través de la frecuencia relativa de los esquemas de junción -elementos que conectan las proposiciones en un discurso- que aparecen en dicho texto. Se recabó un corpus de 200 mensajes de Twitter, proveniente de cuatro cuentas correspondientes a distintas esferas de actividad humana -institucional, comercial, informativa y personal-, para luego hacer un análisis con el fin de encontrar los esquemas de junción presentes en los mensajes, así como elementos progresivamente alejados de lo lingüístico, pero fuertemente vinculados a ello. De esto se obtienen algunos datos estadísticos que luego se someten a interpretación cualitativa, describiendo algunos elementos conservadores de Tradiciones Discursivas en distintas esferas, como la omisión de argumentos y el uso del discurso directo en la esfera informativa, o el diario en la esfera personal. Con este análisis, las cuatro esferas se pueden clasificar en dos grupos, siguiendo la noción de marcos de emisión de Goffman (1981). Los elementos conservadores, aunados a los innovadores, permiten postular la plausibilidad de una Tradición Discursiva nacida en Twitter.

PALABRAS CLAVE: tradiciones discursivas - junción - esquemas de junción esferas - integración sintáctica - complejidad semántica - Twitter.

\section{ABSTRACT}

These lines present some results obtained by testing the hypothesis that suggests the possibility of identifying symptoms of a Discourse Tradition -understood as any

\footnotetext{
1 Juan Ricardo Ubiarco Moya: lingüista. Maestro en Humanidades por la Universidad Autónoma Metropolitana y doctorando en Lingüística.jubiarco@colmex.mx
} 
historically determined mold for communication in a language with its own historicityin a text through the relative frequency of the junction schemes -elements that connect the propositions in a discourse- that appear in a text. A corpus of 200 Twitter messages was collected, from four accounts corresponding to different spheres of human activity -institutional, commercial, informative and personal-, then an analysis is made in order to find the junction schemes present in the messages, as well as the elements out of linguistic scope, but strongly linked to it. From this, some statistical data is analyzed qualitatively, describing some conservative elements of Discourse Traditions in different spheres, such as the omission of arguments and the use of direct discourse in the informative sphere, or the diary in the personal sphere. With this analysis, the four spheres can be classified into two groups, following the notion of emission frameworks by Goffman (1981). The conservative elements together with the innovators, allow to postulate the plausibility of a Discourse Tradition born on Twitter.

KEY WORDS: discourse traditions - junction - junction schemes - spheres syntactic integration - semantic complexity - Twitter.

\section{TRADIÇÃO E INNOVAÇÃO NO TWITTER. UM ENSAIO DE TRADIÇÕES DISCURSIVAS NAS CONTAS MEXICANAS ATRAVÉS DA JUNÇẤO}

\section{RESUME}

Apresentamos alguns resultados obtidos submetendo a experimentação uma hipótese que sugere a possibilidade de identificar sintomas de uma Tradição Discursiva - entendida como todo molde historicamente determinado para a comunicação em uma linguagem com sua própria historicidade - em um texto através da frequência relativa dos esquemas de junção - elementos que conectam as proposições em um discurso - que aparecem no respectivo texto. Se obteve um corpus de 200 mensagens do Twitter, provenientes de quatro contas correspondentes a distintas esferas de atividade humana - institucional, comercial, informativa e pessoal, para logo fazer uma análise com o fim de encontrar os esquemas de junção presentes nas mensagens, assim como elementos progressivamente distantes do linguístico, mas fortemente vinculados a ele. Disso se obteve alguns dados estatísticos que logo se submetem a interpretação quantitativa, descrevendo alguns elementos conservadores de Tradições Discursivas em distintas esferas, como a omissão de argumentos e o uso do discurso direto na esfera informativa, ou o diário na esfera pessoal. Com esta análise, as quatro esferas podem se classificar em dois grupos, seguindo a noção de marcos de emissão de Goffmam(1981). Os elementos conservadores, aunados aos inovadores, permitem postular plausibilidade de uma Tradição Discursiva nascida no Twitter.

PALAVRAS CHAVE: tradições discursivas - junção - esquemas de junção - esferas - integração sintática - complexidade semântica - Twitter. 


\section{Cómo citar el artículo:}

Ubiarco Moya, J. (2020). Tradición e innovación en twitter. Un ensayo de tradiciones discursivas en cuentas mexicanas a través de la junción. [Tradition and innovation in twitter. An essay of discourse traditions in mexican accounts through junction]. Revista de Comunicación de la SEECI, 51, 129-145.

doi: http://doi.org/10.15198/seeci.2020.51.129-145

Recuperado de http://www.seeci.net/revista/index.php/seeci/article/view/591

\section{INTRODUCCIÓN}

Las siguientes líneas plantean un (posible y somero) análisis de varios mensajes tomados del servicio de microblogging Twitter en cuentas mexicanas- fuertemente basado en la hipótesis de Kabatek (2005), que ha parecido plausible -dando el siguiente paso del método científico- poner a prueba: "Los esquemas de junción de un texto -juntores que contiene y frecuencia relativa- son síntomas para determinar la tradición discursiva a la que el texto pertenece" (p. 169).

Toda tradición discursiva (TD) se relaciona con modos de hablar históricamente determinados ${ }^{2}$ y sobre ellos añade elementos de innovación: "[...] siempre la tradición es conservadora e innovadora, y la transformación de una TD en otra siempre consiste en elementos tradicionales y elementos nuevos, como el correo electrónico al inicio partió de la tradición de la carta" (Kabatek, 2007, p. 341). Al ser Twitter un medio de comunicación relativamente nuevo, que sólo ha sido posible dado el desarrollo de Internet y de las redes sociales virtuales, que añaden ciertas posibilidades a la actividad universal del hablar (Coseriu, 1978, p. 45), es posible -e interesante- un estudio de los mensajes de Twitter (tuits), en español mexicano, a la luz de las TD.

El estudio de fenómenos lingüísticos en Internet es un campo bastante amplio desde hace ya varios años; en español, algunas obras ilustrativas son las de Francisco Yus (2010), dedicado a la ciberpragmática, Ana Mancera (2016), que estudia usos subestándares como señas de identidad, Vivas y Ridao (2015), que estudian cortesía en Facebook y Twitter, o Pano y Mancera (2014), sobre los mensajes de políticos españoles en Twitter. En inglés y en el mismo Twitter, hay trabajos de tintes trágicos e interdisciplinarios (O'dea, et al., 2016), acerca de la construcción de comunidad desde una perspectiva sistémico-funcional (Zappavigna, 2011) y más, enmarcados en algún grado en la tuiterología. ${ }^{3}$

\footnotetext{
2 Para Coseriu (1981) toda lengua tiene tres niveles: universal, histórico e individual. Cada uno de ellos requiere de distintos tipos de conocimientos; en el caso del nivel histórico -que es el que aquí se trata-, se requiere conocimiento de reglas idiomáticas — básicamente, la gramática de una lengua histórica particular, aquí el español-y de reglas discursivas, de las cuales se ocupa el estudio de las TD (Koch, 2008).

3 Zimmer (2011) resalta el hecho de que Twitter es "una mina de oro" para investigadores de campos como la lingüística, la psicología y la sociología que buscan datos producidos con gran inmediatez. La conjunción de conocimientos transdisciplinarios que puede servir para estudiar los mensajes en Twitter ha llevado a englobar todo este tipo de estudios bajo el concepto de tuiterología ("twitterology").
} 
Las siguientes líneas están divididas en los siguientes apartados: primeramente se presentan los "Objetivos" de esta pequeña investigación. Luego, en "Método" se anota un poco más ampliamente el concepto de junción, los parámetros cuantitativos y cualitativos atinentes a la conformación del corpus y cómo procedió su análisis. En la "Discusión" se detallan los esquemas de junción y sus frecuencias absoluta y relativa, así como se introducen algunos ejemplos pertinentes extraídos del corpus. Luego, se presentan algunas generalizaciones obtenidas del análisis en busca de elementos de conservación e innovación en los mensajes de Twitter, además de un "acto de contrición" debido a algunas decisiones metodológicas tomadas. Finalmente, son esbozadas algunas escuetas conclusiones sobre los pasos a seguir después de estos primeros resultados.

\section{OBJETIVOS}

Para la realización de este primer experimento, se han planteado dos objetivos: 1) identificar los esquemas de junción en un corpus ex profeso para esbozar algunos indicios de TD en Twitter; 2) relacionar los tuits en el corpus, provenientes de distintas esferas de actividad humana, con mensajes canónicos de cada una de sus respectivas esferas, para sugerir algunos elementos de conservación e innovación.

\section{MÉTODO}

Este estudio parte del concepto de géneros discursivos de Bajtín (1982), "tipos relativamente estables de enunciados" (p. 248) particulares a cada esfera de actividad humana. Dentro de cada género discursivo, es posible encontrar TD, que son "moldes normativos convencionalizados que guían la transmisión de un sentido mediante elementos lingüísticos tanto en su producción como en su recepción" (Oesterreicher apud Kabatek, 2007, p. 338). Se buscó identificar dichos "moldes normativos" con ayuda del concepto de junción ("Junktion") que Wolfgang Raible (1992) propone con fines tipológicos.

La junción denota los "elementos que relacionan los componentes proposicionales en un texto" (Kabatek, 2005, p. 165) y puede analizarse sintáctica y semánticamente. En un eje vertical se considera la integración sintáctica, que tiene siete distintos grados, de mayor a menor: I) yuxtaposición de oraciones; II) anáforas y deixis; III) coordinación; IV) subordinación; V) construcciones con gerundio y participio; VI) grupos (locuciones) preposicionales; VII) preposiciones; y VIII) actantes. En un eje horizontal se localizan distintos niveles de complejidad semántica, que aumenta siguiendo la dirección de la escritura en español: 1) circunstancia ("Begleitumstand"); 2) instrumento ("Instrument"); 3) condición ("Bedingung"); 4) relación temporal ("Zeitrelation"); 5) causalidad ("Kausalität"); 6) "contracausalidad" ("Gegenursache"); y 7) finalidad ("Finalität") (Kabatek, 2005; Raible, 1992, 2001; Renwick, 2006).

Bajtín (1982) no es muy alentador al anotar que el número de géneros discursivos va a la par del número de esferas de la actividad humana. Así que para dar viabilidad a esta investigación, se ha seguido parcialmente la propuesta de Alcoba (2004) para 
delimitar cuatro esferas de la actividad humana: institucional, informativa, comercial y (añadido del autor) personal. Luego se buscó una cuenta de Twitter mexicana por cada una de las esferas: "Presidencia de México" (@PresidenciaMX), el periódico "El Universal" (@El_Universal_Mx), la marca de bebidas "Coca-Cola ${ }^{\circledR}$ México" (@CocaColaMx) y "pedacito de mi vida" (sic) (@carmenfaroo) representan las cuentas de las esferas institucional, informativa, comercial y personal, respectivamente. Se reunió un corpus formado por 50 mensajes de cada una de las cuatro cuentas ( 200 mensajes en total), recabados tomando el 1 de octubre de 2018 como fecha de partida para retroceder en la línea temporal. Fueron omitidos, al recabar el corpus, tuits de otras cuentas compartidos en alguna de las cuentas que se analizaron, así como los "hilos" -cadenas de mensajes de uno o varios usuariospor considerar que poseen algunas características adicionales a las del tuit canónico.

El análisis de los esquemas de junción (EJ) en el corpus se centró en las relaciones entre formas verbales (finitas o no finitas), en tanto que se considera a los verbos, si no como una proposición, sí al menos como funciones proposicionales. ${ }^{4}$ Se hace aquí una aclaración: con el fin de volver más manejable este análisis, se optó por no considerar todos los niveles de complejidad semántica propuestos por Raible (1992), sino únicamente tres: condicionalidad, causalidad y finalidad. Se ha localizado este tipo de relaciones en el corpus para luego anotar el grado de integración sintáctica mediante la cual se manifiestan.

\section{DISCUSIÓN}

Con los parámetros definidos para la búsqueda de EJ -tres niveles de complejidad semántica y ocho de integración sintáctica-, fueron encontrados un total de 102 EJ en los 200 mensajes del corpus. Su distribución y el porcentaje que representan se detallan en la tabla 1.

Tabla 1. Distribución de EJ.

\begin{tabular}{|l|r|r|}
\hline Cuenta & Número de EJ & Porcentaje \\
\hline Presidencia México & 28 & $27.5 \%$ \\
\hline El Universal & 18 & $17.6 \%$ \\
\hline Coca-Cola ${ }^{\circledR}$ México & 36 & $35.3 \%$ \\
\hline pedacito de mi vida & 20 & $19.6 \%$ \\
\hline Total & 102 & $100 \%$ \\
\hline
\end{tabular}

Fuente: elaboración propia.

\footnotetext{
${ }^{4}$ Una función proposicional es una función que puede dar como valores el ser verdadera o falsa -es decir, una proposición - una vez que se saturen los argumentos que requiere (Kandel, 2008).
} 
Aunque el análisis se limitó a tres niveles de complejidad semántica, es evidente que los demás también pueden encontrarse en el corpus; por ejemplo, en (1a) se advierte una relación circunstancial temporal y en (1b) una circunstancial modal.

1a. \#2DeOctubre | Cuando había una liberación todos se alegraban, sin importar si era un amigo cercano o no tuvieran relación alguna. Había felicidad porque unos salían, pero después tristeza para los que se quedaban (ElUniv-8). ${ }^{5}$

1b. Hemos trabajado para mejorar las condiciones de vida de los grupos más vulnerables, reduciendo los niveles de pobreza, en la construcción de un México incluyente: @EPN (Pres-16).

Tabla 2. FA de EJ en el corpus.

\begin{tabular}{|l|r|r|r|}
\hline & 1 & 2 & 3 \\
\hline I & 1 & 12 & 2 \\
\hline II & & & \\
\hline III & 1 & 4 & 2 \\
\hline IV & 8 & 12 & 5 \\
\hline V & & 2 & \\
\hline VI & & 1 & \\
\hline VII & 1 & 7 & 43 \\
VIII & 1 & & \\
\hline
\end{tabular}

Fuente: elaboración propia.

La tabla 2 presenta la frecuencia absoluta (FA) de los EJ encontrados en el corpus. Resalta tras una ligera revisión el número de EJ VII.3 -finalidad expresada mediante preposiciones. En (2a-d) pueden verse ejemplos de este esquema de junción tomados de cada una de las cuentas:

2a. Directivos y personal operativo de diversas Secretarías y dependencias del @GobMx, están desplegados en Sinaloa y Sonora para de manera coordinada con los Gobiernos Estatales, ayudar a la población afectada por las lluvias (Pres-1).

2b. \#AlfombraRoja Arturo López Gavito propuso a @_CarlosRivera a los directivos de Disney para interpretar el tema "Recuérdame" de la película Coco (ElUniv-26).

2c. Aprovecha el medio tiempo de Suecia vs Inglaterra y llévate una de estas increíbles playeras edición limitada de Coca- Cola. iLlama ya y participa para ganar una! (Coca-12).

${ }^{5}$ Los ejemplos se etiquetan con una referencia a la cuenta de la cual se obtuvieron: "Pres" para "Presidencia México", "ElUniv" para "El Universal", "Coca" para "Coca-Cola ${ }^{\circledR}$ México", y "Pedac" para "pedacito de mi vida". El número remite únicamente al número correspondiente en el corpus. Cabe añadir que se ha respetado la escritura de todos los mensajes, únicamente cambiando el tipo de fuente. 
2d. Si me está resultando increíble cómo pasé de querer con todas mis fuerzas verme preciosa en la boda de mis amigas y conseguir un novio para que acompañara a capacidad intelectual para acabar todos mis pendientes de la escuela. Punto para el posgrado. Creo (Pedac-3).

Ahora se presentan y analizan brevemente los EJ de cada cuenta por separado. La gráfica 1 muestra los porcentajes que representan los distintos EJ encontrados en la cuenta "Presidencia México". También en esta cuenta se advierte la concentración de los EJ en el tipo VII.3 -núcleo preposicional en una frase de intepretación final-, dejando en un muy lejano segundo lugar la causalidad expresada mediante yuxtaposición de oraciones (I.2), como en (3), donde lo referido en el primer segmento señalado con cursivas puede interpretarse como causa de lo referido por el segundo.

3. México ha actuado responsablemente en la búsqueda de soluciones a los retos de la migración. Me congratulo de los avances que hemos realizado para llegar a un Pacto Mundial para una Migración Segura, Ordenada y Regular: @EPN

https://www.gob.mx/presidencia \#UNGA (Pres-20).

En la gráfica 2 se anota el porcentaje en que aparecen los EJ identificados en la cuenta de "El Universal". La cual, aunque con muchos menos EJ, sigue la tendencia observada en el caso anterior en tanto al EJ VII.3. En (4) se presenta un ejemplo del EJ IV.2, subordinación significando causalidad, el segundo EJ más común en los tuits tomados de esta cuenta.

4. "Me gustaría decirles a los que se llevaron la computadora que no pueden abrirla porque tienen cosas de trabajo. O sea, no les va a servir de nada", dijo @margaritacumbia (ElUniv-25).

Las gráficas 3 y 4 presentan la misma información que las primeras dos, ahora para las cuentas "Coca-Cola ${ }^{\circledR}$ México" y "pedacito de mi vida, respectivamente. En "Coca-Cola ${ }^{\circledR}$ México" puede verse de nuevo la preeminencia del EJ VII. ${ }^{6}$, seguida ahora por el EJ IV.1, condicionalidad mediante subordinación (5a). Por fin, la cuenta "pedacito de mi vida" rompe la tendencia, inclinándose hacia EJ que manifiestan causalidad mediante yuxtaposición (5b) o mediante subordinación (5c).

\footnotetext{
${ }^{6}$ La "alta" presencia de EJ VII.3 en esta cuenta bien puede deberse a un hecho muy fortuito: la presencia del hashtag —una etiqueta de metadatos- "\#ListosPara" durante la celebración del último campeonato mundial de futbol en Rusia el verano de 2018.
} 




Gráfica 1: EJ en "Presidencia México "ISEEP,

Fuente: elaboración propia.

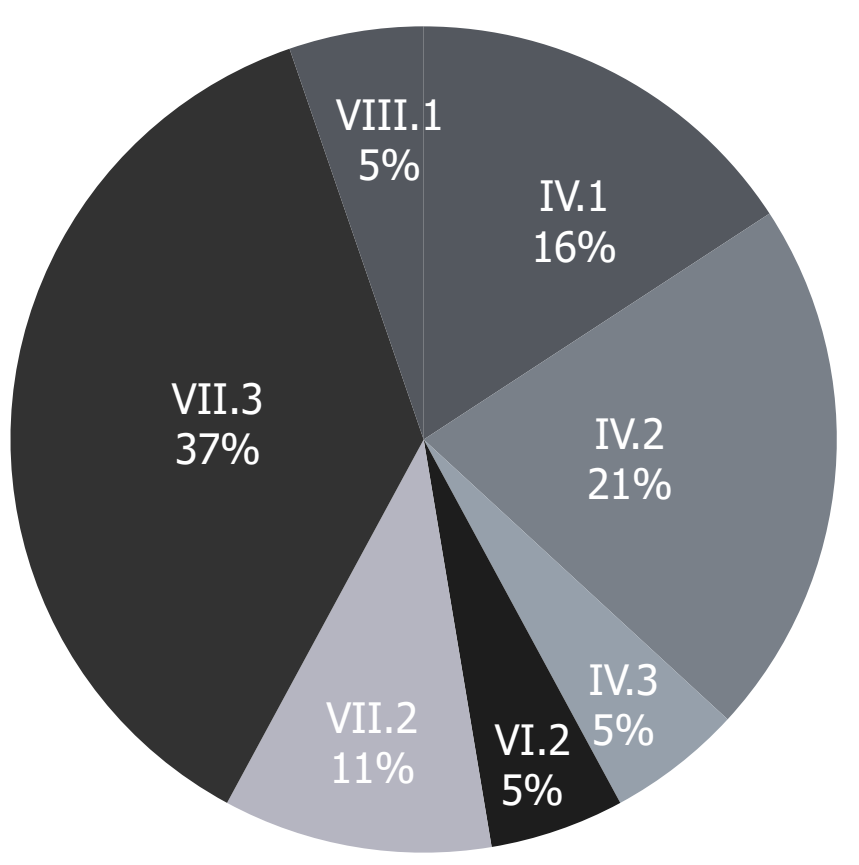

Gráfica 2: EJ en "El Universal ${ }^{\prime \prime S E L E e^{\prime}}$

Fuente: elaboración propia. 
Ubiarco Moya, J. Tradición e innovación en Twitter. Un ensayo de tradiciones discursivas en cuentas mexicanas a través de la junción

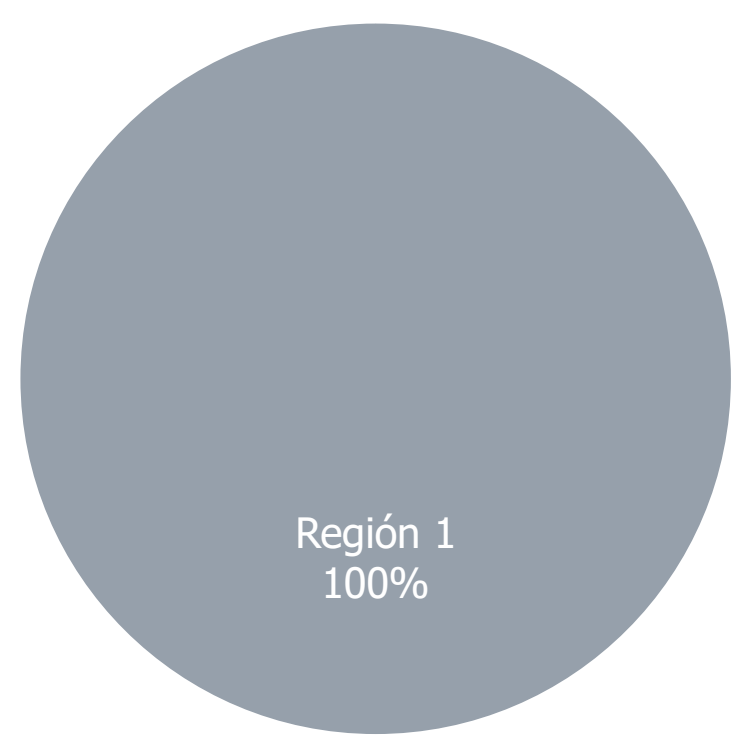

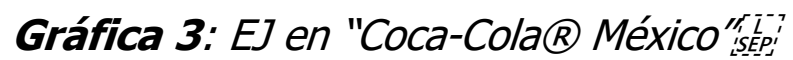

Fuente: elaboración propia.

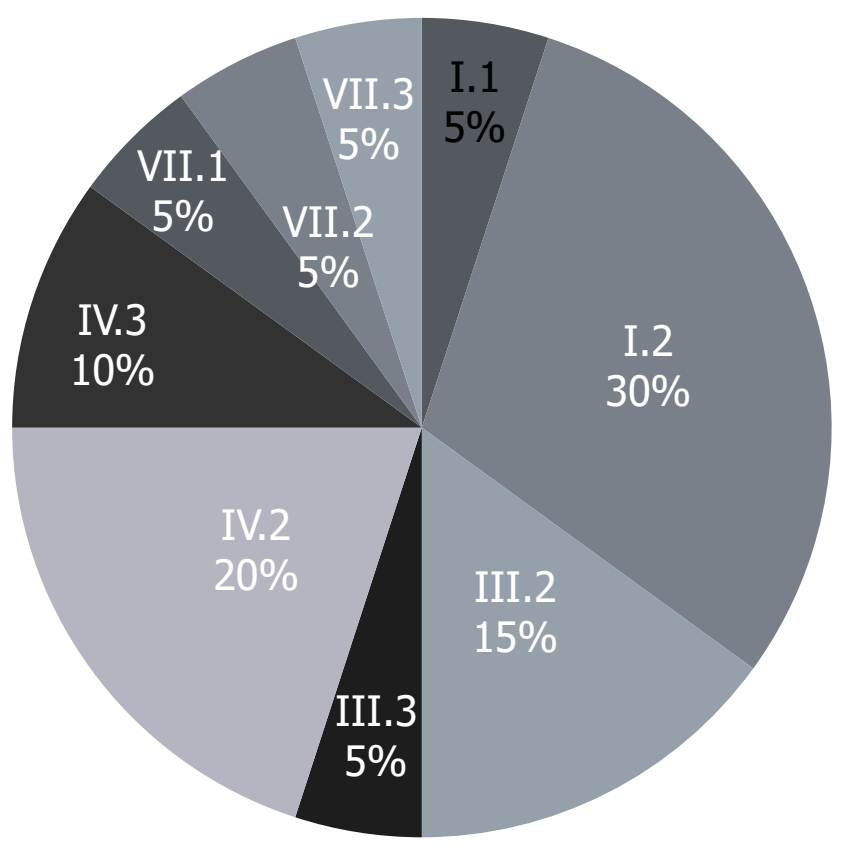

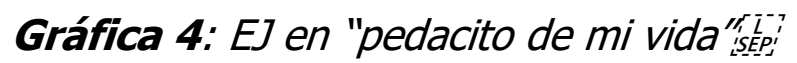

Fuente: elaboración propia.

5a. Estamos \#ListosPara el primer partido de cuartos de final, dos grandes equipos se enfrentan. RT si crees que gana Francia y FAV si vas con Uruguay (Coca-4). 
5b. Mientras comemos pusieron a las Jeans. Esta boda ya ganó mi corona a mejor boda. Sorry not sorry (Pedac-33).

5c. Estaba stalkeando a la novia de un amigo y de pronto vi esta imagen y la verdad es que no sé si estoy más enojada porque le atribuyen la mamarrachada que dijo Charly de STF a Borges o porque mi amigo anda con una niña que cree que es posible que eso lo haya dicho Borges (Pedac-23).

Lo siguiente en este análisis es presentar el cálculo de frecuencia relativa (FR) que refiere Kabatek (2005) como síntomas de una TD. En la tabla 3 se presenta dicho cálculo realizado a los 102 EJ encontrados en el corpus. Luego, la tabla 4 presenta el mismo cálculo, pero anotado para cada una de las cuentas separadamente, estas tablas también muestran la frecuencia absoluta (FA) de los EJ encontrados.

Tabla 3. FA y FR de los EJ en el corpus (1).

\begin{tabular}{|l|r|r|}
\hline EJ & FA & FR \\
\hline I.1 & 1 & 0.00980392 \\
\hline I.2 & 12 & 0.11764706 \\
\hline I.3 & 2 & 0.01960784 \\
\hline III.1 & 1 & 0.00980392 \\
\hline III.2 & 4 & 0.03921569 \\
\hline III.3 & 2 & 0.01960784 \\
\hline IV.1 & 8 & 0.07843137 \\
\hline IV.2 & 12 & 0.11764706 \\
\hline IV.3 & 5 & 0.04901961 \\
\hline V.2 & 2 & 0.01960784 \\
\hline VI.2 & 1 & 0.00980392 \\
\hline VII.1 & 1 & 0.00980392 \\
\hline VII.2 & 7 & 0.06862745 \\
\hline VII.3 & 43 & 0.42156863 \\
\hline VIII.1 & 1 & 0.00980392 \\
\hline Total & 102 & \\
\hline & & \\
\hline
\end{tabular}

Fuente: elaboración propia. 
Ubiarco Moya, J. Tradición e innovación en Twitter. Un ensayo de tradiciones discursivas en cuentas mexicanas a través de la junción

Tabla 4. FA y FR de los EJ en el corpus (2).

\begin{tabular}{|c|c|c|c|c|c|}
\hline EJ (Pres) & FA & $\mathbf{F R}$ & EJ (ElUniv) & FA & FR \\
\hline I. 2 & 3 & 0.10714286 & I. 2 & 0 & 0 \\
\hline I. 3 & 2 & 0.07142857 & I.3 & 0 & 0 \\
\hline IV.1 & 0 & 0 & IV.1 & 3 & 0.16666667 \\
\hline IV. 2 & 1 & 0.03571429 & IV.2 & 4 & 0.22222222 \\
\hline IV. 3 & 1 & 0.03571429 & IV.3 & 1 & 0.05555556 \\
\hline V.2 & 2 & 0.07142857 & V.2 & 0 & 0 \\
\hline VI.2 & 0 & 0 & VI.2 & 1 & 0.05555556 \\
\hline VII.2 & 0 & 0 & VII.2 & 2 & 0.11111111 \\
\hline VII.3 & 18 & 0.64285714 & VII.3 & 7 & 0.38888889 \\
\hline VIII.1 & 1 & 0.03571429 & VIII.1 & 0 & 0 \\
\hline Total & 28 & 1 & Total & 18 & 1 \\
\hline EJ (Coca) & FA & FR & EJ (Pedac) & FA & FR \\
\hline I. 1 & 0 & 0 & I. 1 & 1 & 0.05 \\
\hline I. 2 & 3 & 0.08333333 & I. 2 & 6 & 0.3 \\
\hline III. 1 & 1 & 0.02777778 & III.1 & 0 & 0 \\
\hline III. 2 & 1 & 0.02777778 & III. 2 & 3 & 0.15 \\
\hline III.3 & 1 & 0.02777778 & III. 3 & 1 & 0.05 \\
\hline IV.1 & 5 & 0.13888889 & IV.1 & 0 & 0 \\
\hline IV. 2 & 3 & 0.08333333 & IV. 2 & 4 & 0.2 \\
\hline IV. 3 & 1 & 0.02777778 & IV. 3 & 2 & 0.1 \\
\hline VII.1 & 0 & 0 & VII.1 & 1 & 0.05 \\
\hline VII.2 & 4 & 0.11111111 & VII.2 & 1 & 0.05 \\
\hline VII.3 & 17 & 0.47222222 & VII.3 & 1 & 0.05 \\
\hline Total & 36 & 1 & Total & 20 & 1 \\
\hline
\end{tabular}

Fuente: elaboración propia. 
Los EJ VIII.3 (los más comunes) corresponden a poco más del $64 \%$ de los EJ en los mensajes de "Presidencia México", 39 \% en la cuenta del periódico "El Universal", y al $47 \%$ en "Coca-Cola ${ }^{\circledR}$ México". Por su parte, el EJ I.2 es el más usual en "pedacito de mi vida", con el 30 \% de los EJ; es esta cuenta la que presenta una mayor distribución entre un número más alto de tipos de EJ.

Ahora, se realiza una breve comparación, de naturaleza bastante intuitiva, de los tuits en el corpus con mensajes enmarcados en TD más canónicas dentro de cada una de las esferas de acción analizadas, comenzando con la esfera institucional; los mensajes dentro de ésta se utilizan "en los organismos estatales para coadyuvar a la función de administrar que les compete a las autoridades y facilitar la prestación de servicios a la colectividad" (Potrero, 2013). Los tuits de "Presidencia México" poca semejanza tienen con discursos institucionales como el visto en publicaciones del tipo del Diario Oficial de la Federación, si bien esta publicación corresponde más bien a un discurso de tintes jurídicos, y mucha con la comunicación corporativa, referida a "la totalidad de los recursos de comunicación de los que dispone una organización para llegar efectivamente a sus públicos" (Capriotti, 1999, p. 30) en la tarea de comunicar lo que la corporación realiza; de esta manera podemos ver tuits cuya finalidad es, básicamente, informar sobre las actividades realizadas, principalmente, por el presidente de la república (6a-b).

6a. En el marco de la \#UNGA, el Presidente @EPN participó en el "Panel de Alto Nivel para construir una \#EconomíaOceánica \#Sustentable" http://ow.ly/2sfK30lXgDT

6b. El Presidente @EPN participó en el debate @wef sobre "Desarrollo Sostenible en la Cuarta Revolución Industrial", donde destacó los avances realizados en México para la consecución de los Objetivos de \#DesarrolloSostenible \#ODS \#UNGA @SRE_mx @MexOnu @Agenda2030MX

La misma intención informativa tienen los tuits de la cuenta del periódico "El Universal". En estos mensajes es más fácilmente reconocible el elemento conservador de la TD de los titulares periodísticos. En los tuits del corpus son recurrentes algunos rasgos formales identificados por Nadal (2012) como característicos de los titulares periodísticos. Entre ellos, el presente histórico con omisión de argumentos (7a-b), el uso de discurso directo (7c) o de frases nominales sin determinantes $(7 d)$.

7a. Aseguran que "Black Mirror" vuelve en diciembre http://eluni.mx/thz1dlkw (ElUniv-11).

7b. Condena ONU-DH que no se haya investigado la masacre de Tlatelolco http://eluni.mx/9sejwc46

7c. "No puede hablarse de un traslado de la Secretaría de Cultura si antes no se resuelven las problemáticas que los trabajadores y trabajadoras", comentó Ángeles Medina (ElUniv-29, sic).

7d. \#Cartón "El peso de la ley" de @Helioflores_mex. Aquí más cartones http://eluni.mx/umza1_zp

Probablemente sea la intención informativa la que recorre los mensajes de las dos cuentas anteriores y de la cuenta "Coca-Cola ${ }^{\circledR}$ México" (las tres en las que predomina el EJ VII.3), más que un discurso publicitario en este último caso. Esto se observa en muchos de los tuits en esta cuenta que hacen referencia a eventos de importancia social -como festivales musicales (8a) y competencias deportivas (8b)-; 
empero, es posible encontrar mensajes (8c) con los fines persuasivos característicos del género discursivo de la publicidad (López, 1999).

8a. Aquí el cartel oficial del \#CocaColaFlowFest, inos vemos este 8 de diciembre! (Coca-47).

8b. Bélgica se queda con el tercer lugar y estamos \#ListosPara verlos celebrar la mejor posición en su historia en la Copa Mundial de la FIFA (Coca-25).

8c. Una familia, dos equipos, una mesa. No importan tus colores, el futbol sabe mejor con botana y Coca-Cola \#SienteEISabor (Coca-49).

Por último, el relativo equilibrio en la distribución de los EJ en "pedacito de mi vida" bien puede ser sintomático del palimpsesto que representan los mensajes de la esfera personal. De acuerdo con Yus (2010), la finalidad de Twitter es que el usuario, mediante sus mensajes, responda a preguntas como "¿Qué estás haciendo?" o "¿Qué pasa?", caso que se muestra en (9a). En esta cuenta también es posible encontrar expresión de pensamientos (9b) y estados de ánimo (9c); referencia a noticias, con un agregado "ecoico" -alguna valoración personal del suceso- (9d) y, en el mismo tenor "ecoico", se retoman fragmentos de poemas (9e) o canciones (9f). Ante tal heterogeneidad, sólo es posible encontrar un lejano símil en el diario personal, los mensajes en una cuenta de Twitter personal son, de alguna manera, una parte de la autobiografía de quien los escribe.

9a. Leí 'princesa' donde decía 'preciosa'. Que si esto es el amor, que si esto es el amor(?) (Pedac-40).

9b. Los últimos sucesos me han llevado a pensar mucho en una de las malísimas películas de Karla Souza donde dicen que el amor debe ser fácil y creo que sí debe ser fácil y si no es así entonces no es ahí (Pedac-22).

9c. Muerta por dentro pero dama por fuera (Pedac-13).

9d. Qué notición lo de los recorridos en la Casa de los enanos en la Juárez. What a time to be alive y ser poblano, la vdd.

9e. «así/sin intenciones misteriosas sé que voy a elegir de buena gana de mi viejo jardín sólo tus rosas de las altas ventanas tu ventana de los signos del mar tu mar de cosas y de todo el amor/ tu amor/mengana» (Pedac-9).

9f. Amanecí bien y dicen los del coro todo un caballero no salta al vacío desde un piso primero no salta al vacío desde un piso primero (Pedac-1).

Para concluir esta sección, debe -mínimamente- mencionarse el papel de los hashtags y de los códigos de usuario (introducidos por una arroba), tal vez uno de los elementos innovadores más prominentes del uso de la lengua en redes sociales por Internet. En un tuit, un hashtag o el código de un usuario pueden interpretar un papel en dos dimensiones: una gramatical, en tanto elemento de las estructuras oracionales concretadas en un enunciado, y una "ciberpragmática", enlazando el enunciado específico con toda la conversación cuyo tema el hashtag etiqueta o con el usuario mencionado en un tuit y cuyo código remplaza un nombre propio. Queda esto ejemplificado en (10a-b).

10a. ¿Ya viste \#ConLosDeEnfrente? El programa de análisis y debate de @El_Universal_Mx y @Milenio. Con @hdemauleon, @apontedavid, @elisaalanis, @jpbecerraacosta, @azucenau y Carlos Marín, conducido por @hzamarron. Todos los miércoles a las 20:30 horas (ElUniv-27).

10b. En la \#UNGA el Presidente @EPN presentará las prioridades de México en materia de política exterior, así como un balance de los logros en materia multilateral alcanzados durante su Administración. http://ow.ly/P7nl30lXVD5 @SRE_mx \#MéxicoGlobal (Pres-12). 
Las FR de los EJ analizados permiten una clasificación de las cuatro cuentas en dos grupos, basados en la descomposición que Goffman (1981) realiza de la noción de "hablante" en tres papeles: "animator", autor y "principal".7 En el caso de tres cuentas, "Presidencia México", "El Universal" y "Coca-Cola ${ }^{\circledR}$ México", es esperable que cada uno de estos papeles esté desempeñado por (conjuntos de) individuos distintos, uno para cada papel. Por otra parte, en una cuenta personal, como "pedacito de mi vida", la situación normal es que los tres papeles recaigan sobre un mismo individuo, excepto, tal vez, en casos como los recién vistos (9e-f), en los que el papel de autor no corresponde al titular de la cuenta personal.

Esta situación sigue la misma línea que el análisis de EJ mostrado en la sección anterior, que arroja una mayor presencia de EJ VII.3 en las primeras tres cuentas, así como una ligera tendencia a los EJ I.2 en la cuenta personal, que muestra también una mayor distribución de EJ en los mensajes. Cabe confesar que la búsqueda de EJ no ha sido lo exitosa que se pretendió; probablemente los niveles de complejidad semántica a los que se restringió el análisis restringieron también los resultados de la búsqueda. A ello se suma que, en español, podría argumentarse que dichas relaciones semánticas se cristalizan canónicamente en construcciones más o menos específicas: una preposición "para" si se desea indicar finalidad, una conjunción "si" para marcar condicionalidad o la conjunción "porque" señalando causa. Empero, sí ha sido posible observar algunos patrones en los tuits que apuntan hacia algunos elementos de conservación en las TD, siendo el caso más claro la relación de los tuits del periódico "El Universal"con los titulares de prensa.

\section{CONCLUSIONES}

En este último apartado se sugiere lo que parece la vía más fértil para continuar esta investigación. Una versión más acabada y ambiciosa que la de este bosquejo podría continuar replicando el análisis de junción, de forma más completa, en mensajes tradicionales de cada esfera de acción y los parcialmente analizados aquí, lo cual implicaría la elaboración de un corpus obtenido de textos como periódicos, anuncios publicitarios y diarios personales no necesariamente limitados al español mexicano. Ello permitiría un estudio contrastivo de EJ en los textos de distintas esferas con un mucho mayor sostén empírico para probar la hipótesis de Kabatek (2005), ya mencionada, que ha guiado esta pequeño trabajo.

Pese al pequeño avance que este texto podría parecer en relación con el proyecto esbozado renglones arriba, sí ha podido percibirse una distinción, a través de los EJ empleados en los distintos textos, entre cuentas de Twitter mexicanas pertenecientes a distintas esferas de acción. Este hallazgo lingüístico correlaciona, en

\footnotetext{
${ }^{7}$ Goffman (1981) analiza la categoría de "hablante" de una forma más realista, descomponiéndola en tres categorías. Así, distingue un "animator" —encargado de la emisión física del mensaje-, un autor -detrás de la selección de ideas, emociones, sucesos, etc., y los elementos lingüísticos que los codifican- y un "principal" - que corresponde al dueño de la posición ideológica representada en el mensaje.
} 
cierta medida y hasta aquí, a modo de hipótesis, con distintas formas de producción de los textos, vistas con ayuda de las nociones analíticas propuestas por Goffman (1981).

Una última nota acerca de la innovación en las TD: el estudio de los elementos de innovación en las TD pasaría por un análisis más concienzudo -y ayudado por herramientas teóricas de las que no se ha dispuesto aquí- del papel de los hashtags y de los elementos audiovisuales en la búsqueda de una mejor definición de las características de una posible TD nacida en Twitter ( $y$, de forma un poco general, con toda posible TD en los usos de la lengua en Internet). Algunos elementos sintomáticos de innovación en los tuits recaen fuertemente en fenómenos progresivamente más alejados de lo estrictamente lingüístico, por lo que van más allá de los límites impuestos a esta investigación y los intrínsecos al investigador. Puede mencionarse en primer lugar la función gramatical y "ciberpragmática" de los hashtags y de los códigos de usuario. El tuit también se ve enriquecido por la posibilidad que el servicio de Twitter ofrece para acompañar el texto con elementos audiovisuales, cuya interacción con lo lingüístico es causa, por ejemplo, de omisión de los nombres de personajes involucrados en el evento referido por el tuit. La misma plataforma ofrece la oportunidad para jugar con elementos tipográficos creando mensajes que bien recuerdan la obra de Guillaume Apollinaire.

Así, entre las tradiciones del diario personal, el titular periodístico o el mensaje publicitario y el juego con códigos de soporte (audio)visual, están algunos de los elementos de innovación y conservación en Twitter.

\section{REFERENCIAS}

Alcoba, S. (2004). Cortesía e imagen en la lengua de Internet. En D. Bravo y A. Briz (Eds). Pragmática sociocultural: estudios sobre el discurso de cortesía en español (pp. 357-370). Barcelona: Ariel.

Bajtín, M. (1982). Estética de la creación verbal. México: Siglo XXI.

Capriotti, P. (1999, agosto). Comunicación corporativa. Una estrategia de éxito a corto plazo. Reporte C\&D-Capacitación y Desarrollo, 13, 30-33.

Coseriu, E. (1978). Sincronía, Diacronía e Historia. El problema del cambio lingüístico. Madrid: Gredos.

Conseriu, E. (1981). Lecciones de lingüística general. Madrid: Gredos.

Goffman, E. (1981). Footing. En Forms of Talk. Pennsylvania: University of Pennsylvania Press.

Kabatek, J. (2005). Tradiciones Discursivas y cambio lingüístico. Lexis, XXIX(2), 151177. 
Ubiarco Moya, J. Tradición e innovación en Twitter. Un ensayo de tradiciones discursivas en cuentas mexicanas a través de la junción

Kabatek, J. (2007). Las tradiciones discursivas entre conservación e innovación. Rivista di filologia e letterature ispaniche, 10, 331-345.

Kandel, A. (2008). Discrete Mathematics for Computer Scientists and Mathematicians. New Jersey: Prentice Hall.

Koch, P. (2008). Tradiciones discursivas y cambio lingüístico: el ejemplo del tratamiento vuestra merced en español. En J. Kabatek (Ed.) Sintaxis histórica del español y cambio lingüístico. Nuevas perspectivas desde las Tradiciones Discursivas (pp. 53-87). Madrid: Iberoamericana.

López, A. (1999). Esencia retórica del mensaje publicitario. Tópicos. Revista de Filosofía, 17, 129-152.

Mancera, A. (2016). Usos lingüísticos alejados del español normativo como seña de identidad en las redes sociales. Bulletin of Spanish Studies, 93(9), 1469-1493.

Nadal, J. (2012). Rasgos formales de los titulares periodísticos: notas sobre diez diarios del ámbito hispánico. Acta Poética, 33(1), 173-195.

O'dea, B., Larsen, M., Batterham, P., Calear, A. \& Christensen, H. (2016, marzo). Talking suicide on Twitter: Linguistic style and language processes of suiciderelated posts. European Psychiatry, 33, S274-S274.

Pano, A. y Mancera, A. (2014). La 'conversación' en Twitter: las unidades discursivas y el uso de marcadores interactivos en los intercambios con parlamentarios españoles en esta red social. Estudios de lingüística del español, 35, 234-268.

Potrero, H. (2013, 23 de febrero). La comunicación oficial [Mensaje publicado en blog]. Consultado el 20 de noviembre de 2018 de http://comunicacionofial.blogspot.com/

Raible, W. (1992). Junktion: eine Dimension der Sprache und ihre Realisierungsformen zwischen Aggregation und Integration. Heidelberg: Carl Winter-Universitätsverlag.

Raible, W. (2001). Linking clauses. En M. Haspelmath, E. König y W. Oesterreicher (Eds.) Language tipology and language universals. An international handbook (pp. 590-617). Berlín, New York: Walter de Gruyter.

Renwick, R. (2006). En torno a la junción como dimensión universal del lenguaje. Lexis, XXX(2), 273-290.

Vivas, J. y Ridao, S. (2015). Estrategias de (des)cortesía en redes sociales: análisis comparativo de facebook y Twitter. Sintagma: revista de lingüística, 2, 773-87. 
Ubiarco Moya, J. Tradición e innovación en Twitter. Un ensayo de tradiciones discursivas en cuentas mexicanas a través de la junción

Yus, F. (2010). Ciberpragmática 2.0. Nuevos usos del lenguaje en Internet. Barcelona: Ariel.

Zimmer, B. (2011, 29 de octubre). Twitterology: A New Science? The New York Times. Consultado el 15 de noviembre de 2018 de https://www.nytimes.com/2011/10/30/opinion/sunday/twitterology-a-newscience.html

Zappavigna, M. (2011). Ambient affiliation: A linguistic perspective on Twitter. New Media and Society, 13(5), 788-806.

\section{AUTOR:}

\section{Juan Ricardo Ubiarco Moya}

Licenciado en Letras Españolas por la Universidad de Guanajuato y maestro en Humanidades por la Universidad Autónoma Metropolitana. Actualmente trabajo en mi tesis doctoral en El Colegio de México A. C. Me intereso principalmente en temas de semántica y pragmática.

jubiarco@colmex.mx

Orcid ID: https://orcid.org/0000-0003-3472-9978 